\title{
Deciphering upper respiratory tract microbiota complexity in healthy calves and calves that develop respiratory disease using shotgun metagenomics
}

\author{
Natália C. Gaeta, ${ }^{*}$ Svetlana F. Lima,† Andre G. Teixeira, † Erika K. Ganda,† Georgios Oikonomou,†‡ \\ Lilian Gregory, ${ }^{*}$ and Rodrigo C. Bicalhot ${ }^{1}$ \\ *Department of Medical Clinic, School of Veterinary Medicine and Animal Science, University of São Paulo, São Paulo, Brazil 05508-270 \\ †Department of Population Medicine and Diagnostic Sciences, Cornell University, Ithaca 14853, NY \\ ‡Department of Epidemiology and Population Health, Institute of Infection and Global Health, University of Liverpool, Neston CH64 7TE, \\ United Kingdom
}

\begin{abstract}
Bovine respiratory disease $(\mathrm{BRD})$ is a multifactorial disorder responsible for severe economic losses in dairy and feedlot herds. Advances in next-generation sequencing mean that microbial communities in clinical samples, including non-culturable bacteria, can be characterized. Our aim was to evaluate the microbiota of the upper respiratory tract of healthy calves and calves with BRD using whole-genome sequencing (shotgun metagenomics). We performed deep nasopharyngeal swabs on 16 Holstein heifer calves (10 healthy and 6 diagnosed with BRD during the study) at 14 and 28 d of life in 1 dairy herd near Ithaca, New York. Total DNA was extracted, and whole-genome sequencing was performed using the MiSeq Illumina platform (Illumina Inc., San Diego, CA). Samples included 5 predominant phyla: Proteobacteria, Firmicutes, Actinobacteria, Bacteroidetes, and Tenericutes. At the genus level, we observed differences between groups for Pseudomonas spp. At the species level, Mannheimia haemolytica was the most abundant bacterium detected. We detected significant differences between groups of calves in the relative abundance of Pseudomonas fluorescens. Pasteurella multocida was among the 20 most abundant species, and Moraxella catarrhalis, commonly associated with pneumonia in humans, was detected in all groups. Analysis of resistance to antibiotics and compounds profiling revealed differences in cobalt-zinc-cadmium resistance. Further research to elucidate the role of Moraxella catarrhalis in BRD is warranted. Genes that were resistant to cobalt-zinc-cadmium, observed mostly in calves with $\mathrm{BRD}$, might be associated with difficulties in antibiotic treatment.
\end{abstract}

Received May 27, 2016.

Accepted October 27, 2016.

${ }^{1}$ Corresponding author: rcb28@cornell.edu
Key words: bovine respiratory disease, whole-genome sequencing, calf

\section{INTRODUCTION}

Bovine respiratory disease (BRD) is a multifactorial disorder (Gershwin et al., 2015) responsible for severe economic losses in dairy and feedlot herds, as a result of its high morbidity and mortality rates (USDA, 2013). Furthermore, BRD has substantial long-term consequences, negatively affecting growth, reproductive performance, and longevity (Waltner-Toews et al., 1986; Virtala et al., 1996a,b; Cernicchiaro et al., 2013). It has been estimated that $7 \%$ of total production costs in feedlots in the United States are related to BRD (Griffin, 1997).

Animals' upper respiratory tract normally harbors a variety of microorganisms that coexist in a harmonious state (Bosch et al., 2013). However, certain events can lead to imbalances in this microbiome and eventually to respiratory disease. In cattle, viral infections, stress (i.e., transportation and mixing), and environmental conditions (i.e., stocking density, climate, and temperature) are important predisposing factors to this imbalance, leading to respiratory tract bacterial infections facilitated by immunosuppression in the host (Griffin et al., 2010). The most commonly described bacteria in BRD are Mannheimia haemolytica, Pasteurella multocida, Histophilus somni, and Mycoplasma spp., particularly Mycoplasma bovis (Aebi et al., 2015; Holman et al., 2015a). These species are part of the upper respiratory tract microbiome. Similarly, humans' upper respiratory tract serves as a reservoir of commensal bacteria such as Haemophilus influenza, Streptococcus pneumoniae, Staphylococcus aureus, and Moraxella catarrhalis, which can in certain cases invade the lungs and cause pneumonia (Bosch et al., 2013).

Culture-dependent techniques are often used to determine the microorganisms associated with respiratory disease. However, these techniques have limitations, 
the main one being that several important microbes are difficult, if not impossible, to culture in the laboratory (Sharpton, 2014). Advances in next-generation sequencing have allowed for in-depth investigation of the microbiomes in clinical samples, determining their taxonomic composition and including unculturable species. Different from 16S metataxonomics, wholegenome sequencing (shotgun metagenomics) provides taxonomic descriptions of microbial communities and important information about gene prediction, protein diversity, and functional profile (Sharpton, 2014).

Studies using 16S metataxonomics to evaluate the nasal, nasopharyngeal, and lung microbiota in feedlot cattle have already been published (Holman et al., 2015b; Timsit et al., 2016). However, to the best of our knowledge, shotgun metagenomics has not been used to investigate BRD. Our aim was to characterize the microbiota of the upper respiratory tract of healthy calves and calves with BRD using whole-genome sequencing, to identify bacteria that may be related to the development of BRD, and to describe their functional profile.

\section{MATERIALS AND METHODS}

The present study was conducted at the Department of Population Medicine and Diagnostic Science, College of Veterinary Medicine, Cornell University, New York, from August 2015 to January 2016. We selected 16 Holstein heifer calves from a larger cohort of 174 animals that were included in a different experiment (Lima et al., 2016). This larger-scale study was conducted on a commercial dairy farm located near Ithaca, New York, that at the time had about 7,000 animals in total (3,500 lactating Holsteins cows) and approximately 600 preweaning calves raised in groups of 25 per pen. All procedures were carried out in agreement with the guidelines of the Institutional Animal Care and Use Committee of Cornell University (protocol 2013-0076).

\section{Animals and Facilities}

Pregnant cows at stage 1 or 2 of parturition were transferred from the close-up free-stall barn into 2 maternity pens (400 $\mathrm{m}^{2}$, deep-bedded). After parturition, calves were removed from the maternity pen and placed in a newborn pen bedded with dry sawdust and heated with heating lamps during the winter months. Feeding and pen characteristics were described by Lima et al., (2016). Calves used in this study were allocated to 9 different pens (25 calves per pen), and all calves remained in the same pen from day 1 of life until they were fully weaned (approximately $65 \mathrm{~d}$ of life). The age of calves in a pen did not differ by more than $3 \mathrm{~d}$.

\section{Case Definition}

The definition for detection of bovine respiratory disease was the presence of 2 or more of the following clinical signs: cough, rectal temperature $>39.5^{\circ} \mathrm{C}$, respiratory rate $>40$ breaths $/ \mathrm{min}$, increased cranioventral lung sounds, or wheezes. Two dedicated farm employees with over 10 years of experience and trained by Cornell University veterinarians (Ambulatory and Production Medicine department), were responsible for overseeing the calf facility and detecting BRD. An experienced veterinarian member of our research team performed a second confirmatory physical examination. Calves diagnosed with BRD were treated according to standard farm protocols (Resflor Gold, Merck Animal Health, Madison, NJ). Treatment was administered on the day of the diagnosis.

\section{Deep Nasopharyngeal Swab Collection}

As part of the larger-scale observational study, deep nasopharyngeal swabs were collected from a cohort of 174 Holstein heifer calves on d 3, 14, 28, and 35 of their life using a 20-cm DNA-free sterile swab (Puritan Medical Products, Guilford, ME) covered by a thin sterile plastic sheath, as described by Lima et al. (2016). Samples were kept on ice until they were transferred to the laboratory and then stored at $-20^{\circ} \mathrm{C}$ until further processing. For the present study, we used deep nasopharyngeal swabs collected at 14 and $28 \mathrm{~d}$ of life from 10 calves that remained healthy throughout the preweaning period (randomly selected from all calves that remained healthy throughout the study) and 6 calves that showed clinical signs of BRD preweaning (randomly selected from all calves that showed signs of BRD during the study).

On average, BRD was diagnosed on d 19. The earliest day of diagnosis was 14 , and the latest was 26 . We therefore had 4 groups of samples: 10 obtained from healthy calves at $14 \mathrm{~d}$ of life (healthy-14d); 6 obtained at $14 \mathrm{~d}$ of life from calves that developed BRD between 14 and $26 \mathrm{~d}$ of life (BRD-14d); 10 obtained from healthy calves at $28 \mathrm{~d}$ of life (healthy-28d); and 6 obtained at $28 \mathrm{~d}$ of life from calves that developed BRD between 14 and $26 \mathrm{~d}$ of life (BRD-28d).

\section{DNA Extraction and Whole-Genome Sequencing}

We extracted total DNA from deep nasopharyngeal samples by adding $1.5 \mathrm{~mL}$ of DNA-free water into a microcentrifuge tubes containing cotton swabs, followed by mechanical disruption using a Mini-Beader-8 (Biospec Products, Battersville, OK). Swabs were removed from 
the tubes, and the remaining liquid was centrifuged at $13,000 \times g$ for $10 \mathrm{~min}$ at room temperature. The DNA was extracted from pellets using a PowerSoil DNA Isolation Kit (MO BIO Laboratory Inc., Carlsbad, CA) according to the manufacturer's protocol (https:// mobio.com/media/wysiwyg/pdfs/protocols/12888.pdf; Lima et al., 2016).

Samples were diluted by adding UltraPure Water (Invitrogen, Waltham, MA) until a concentration of $2.5 \mathrm{ng} / \mu \mathrm{L}$ was reached, measured using a Qubit fluorometer (Thermo Fisher Scientific, Waltham, MA). We prepared a sample library for cluster generation and sequencing using a Nextera DNA Sample Preparation Kit (Illumina Inc., San Diego, CA) according to the manufacturer's protocol (http://support.illumina.com/ content/dam/illumina-support/documents/documentation/chemistry_documentation/samplepreps_nextera/nexteradna/nextera-dna-library-prep-referenceguide-15027987-01.pdf).

Briefly, a mixture of $5 \mu \mathrm{L}$ of DNA (4 nmol) and 0.2 $N \mathrm{NaOH}$ was vortexed and centrifuged to $280 \times g$ for $1 \mathrm{~min}$. The mixture was incubated for $5 \mathrm{~min}$ to allow DNA denaturation. Following DNA denaturation, 990 $\mu \mathrm{L}$ of hybridization buffer was added to the diluted DNA to obtain a $2 \mathrm{n} M$ library. We ensured quality control for cluster generation, sequencing, and alignment by adding PhiX Control $4 \mathrm{n} M$ (Illumina Inc.), prepared using $2 \mu \mathrm{L}$ of $10 \mathrm{n} M$ PhiX library and $3 \mu \mathrm{L}$ of $10 \mathrm{n} M$ Tris-Cl pH8.5 with $0.1 \%$ Tween 20 . We performed pair-end sequencing using a MiSeq Reagent Kit v3 (600 cycles) in the Illumina MiSeq platform.

\section{Bioinformatics and Statistical Analysis}

Raw data files were de-multiplexed and converted to fastq files using Casava v.1.8.2 (Illumina, Inc.). Fastq files were concatenated and uploaded to the Metagenome Rapid Annotation Using Subsystems Technology (MG-RAST) server to determine the relative abundance of microbiota at the phylum, genus, and species levels, and predict metabolic characteristics. In the MG-RAST pipeline, sequences were subjected to quality control, including dereplication (removal of sequences produced by sequencing artifacts), removal of host-specific species sequences (Bos taurus, UMD v3.0), ambiguous base filtering (removal of sequences with $>5$ ambiguous base pairs), and length filtering (removal of sequences with a length $>2$ standard deviations from the mean). To reduce the effect of experimental noise/error, we used the normalized data option in MG-RAST. Normalization was performed using a $\log 2$-based transformation $[\log 2(\mathrm{x}+1)]$, followed by standardization within each sample and linear scaling across all samples. We used a nonredundant multisource protein annotation database (M5NR; http://metagenomics.anl.gov/) as the annotation source for organism classification. Organism abundance was analyzed using a best-hit classification approach with a maximum evalue of $1 \times 10^{-5}$, a minimum identity cutoff of $60 \%$, and a minimum alignment length cutoff of 15 . We used the SEED (http://metagenomics.anl.gov/) as the annotation source for predicted metabolic profiles. We performed predicted metabolic abundance analysis using hierarchical classification, also with a maximum e-value of $1 \times 10^{-5}$, a minimum identity cutoff of $60 \%$, and a minimum alignment length cutoff of 15 .

We extracted normalized abundances of phyla, genera, species, and functional profile elements from MGRAST and analyzed using ANOVA in conjunction with Tukey's test for multiple comparisons using JMP Pro 12 (SAS Institute Inc., Cary, NC). We also constructed heatmaps for phyla distribution in JMP Pro 12. Results are presented as least squares means followed by the standard error of the mean. Dependent variables in our statistical analyses were the normalized relative abundances of microbial types; the independent variable was health status and sampling time (BRD-14d, BRD-28d, healthy-14d, healthy-28d). We performed comparisons to identify potential bacteria and functional features that may have been related to the development of BRD during the period when calves are more predisposed to it (Virtala et al., 1996a). Variables with $P<0.05$ were considered statistically different. Variables with $0.1<$ $P<0.05$ were considered to be statistical tendencies.

We deposited shotgun sequencing data in MG-RAST under the following accession numbers: 4678805.3, 4678806.3, 4678807.3, 4678808.3, 4678809.3, 4678810.3, 4678811.3, 4678812.3, 4678813.3, 4678814.3, 4678815.3, 4678816.3, 4678817.3, 4678818.3, 4678819.3, 4678820.3, 4678821.3, 4678822.3, 4678823.3, 4678824.3, 4678825.3, 4678826.3, 4678827.3, 4678828.3, 4678829.3, 4678830.3, 4678831.3, 4678832.3, 4678833.3, 4678834.3, 4678835.3, 4678836.3 .

\section{RESULTS}

\section{Sequencing Results}

Sequencing of deep nasopharyngeal samples generated 21,155,233 sequences (mean number of sequences per sample: 661,101; median: 638,404; range: 383,190 994,172); 2,307,911,887 base pairs (mean: 72,122,246.46; median: $73,160,664$; range: $40,628-100,847,895)$; 310,656 high-quality sequences (mean: 9,708; median: 9,330; range: 4,954-16,210), and an average length of 111.1 (median: 115.5; range: 74-138). 


\section{Phylum}

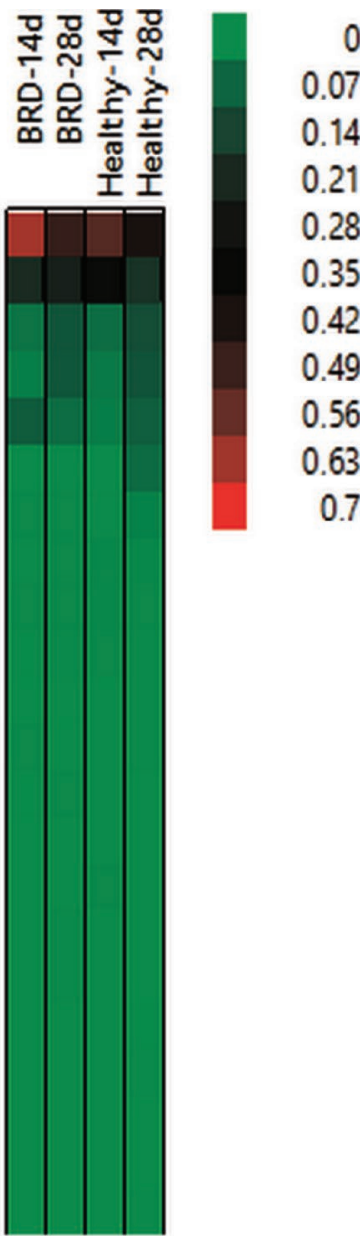

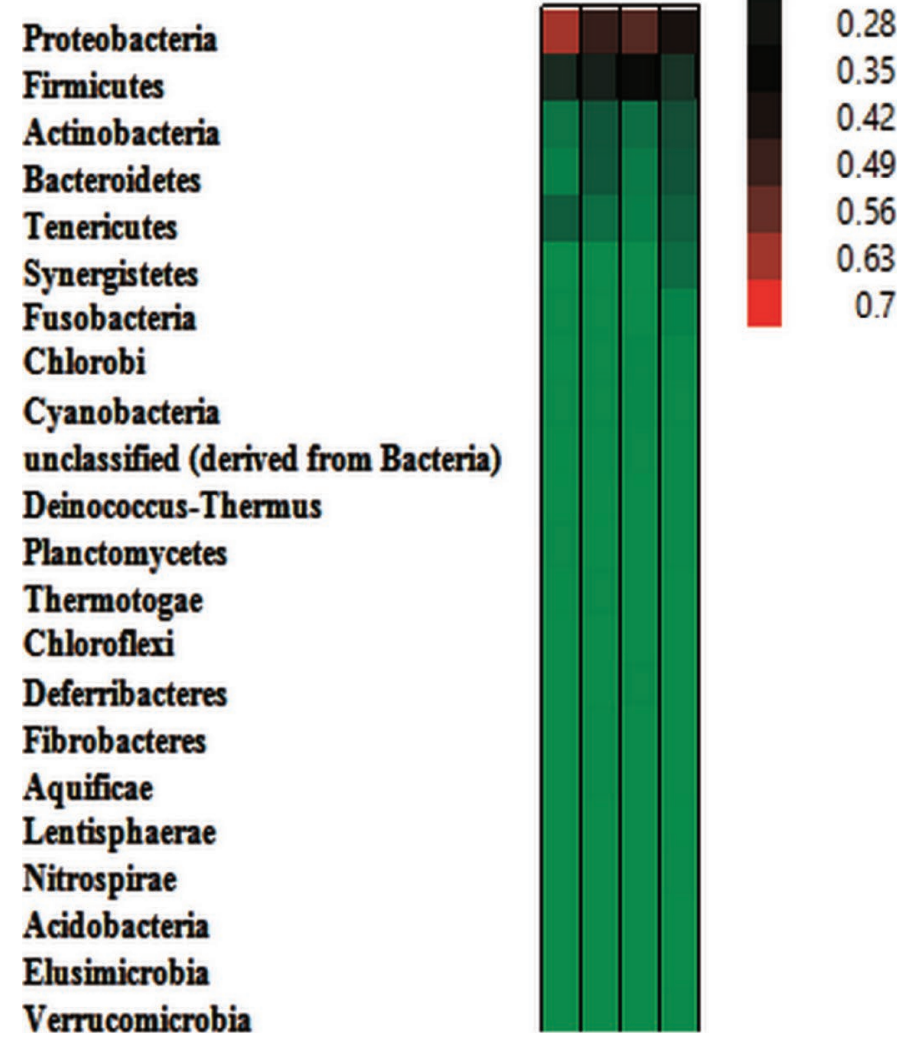

Figure 1. Heat map demonstrating the relative abundance of different phyla in deep nasopharyngeal swab samples obtained from healthy calves at $14 \mathrm{~d}$ of life (healthy-14d); calves that developed bovine respiratory disease (BRD) between 14 and $26 \mathrm{~d}$ of life (samples obtained at $14 \mathrm{~d}$ of life, BRD-14d); healthy calves at $28 \mathrm{~d}$ of life (healthy-28d); and calves that developed BRD between 14 and $26 \mathrm{~d}$ of life (samples obtained at $28 \mathrm{~d}$ of life, BRD-28d). Color version available online.

\section{Nasopharyngeal Microbiota}

In total, we detected 22 phyla, but we identified 5 predominant phyla as the most abundant in all groups: Proteobacteria, Firmicutes, Actinobacteria, Bacteroidetes, and Tenericutes (Figure 1). Together, these major phyla comprised 96, 97, 95, and $80 \%$ of the detected phyla in BRD-14d, BRD-28d, healthy-14d, and healthy28d calves, respectively. Within Proteobacteria, Pseudomonas spp. were the most abundant (11\%), of which Pseudomonas fluorescens was the most abundant. This phylum consisted mainly of Pseudomonas spp., Psychrobacter spp., Mannheimia spp., Actinobacillus spp., Enhydrobacter spp., Escherichia spp., Acinetobacter spp., Haemophilus spp., and Moraxella spp.
We observed a significant difference in phyla relative abundance between groups for Bacteroidetes $(P<$ 0.05). A comparison of the Bacteriodetes mean relative abundance between health status and sampling time point revealed significant differences between healthy28d and healthy-14d samples $(P<0.05)$.

Analysis at the genus level revealed 20 dominating genera (Figure 2). The most important genera related to respiratory diseases, such as Mycoplasma spp., Mannheimia spp., and Pasteurella spp., were detected in all groups (Figure 2), and we observed numeric (although statistically not significant) differences.

Figures 3 and 4 show the relative abundances of the 20 most abundant bacteria (species-level information) detected in deep nasopharyngeal samples from healthy calves and calves that developed BRD. The relative abundance of Pseudomonas fluorescens was $0.07 \pm 0.04$ for BRD-14d, $0.002 \pm 0.002$ for BRD-28d, $0.01 \pm 0.005$ for healthy-14d, and $0.002 \pm 0.001$ for healthy-28d $(P$ $<0.05)$. Although no significant differences were detected between the main bacteria related to pneumonia in calves and health status [Mannheimia haemolytica $(P=0.60)$ and Pasteurella multocida $(P=0.83)]$, these pathogens were within the 20 most abundant species, unlike Histophilus somni and Mycoplasma bovis (Figures 3 and 4). Mannheimia haemolytica was the most abundant bacterium, and its relative abundance was numerically increased in both BRD-28d (0.06 \pm 0.03$)$ and healthy-28d calves $(0.07 \pm 0.02)$ compared to BRD-14d and healthy-14d calves. On the other hand, the relative abundance of Pasteurella multocida was low in all 4 groups (Figure 4). We detected Moraxella catarrhalis in all groups of calves. The relative abundance of the bacteria most commonly associated with pneumonia (Mannheimia haemolytica, Pasteurella multocida, Histophilus somni, and Mycoplasma bovis) are also presented in Figure 5.

The proportion of calves with a positive detection of these known pathogens is presented in Table 1 . We detected Mannheimia haemolytica in most of the studied calves $(78.12 \%)$. On the other hand, we observed lower proportions of Mycoplasma bovis (18.75\%). We detected Mycoplasma bovis in $16.7 \%$ of the calves in both BRD groups and in $40 \%$ of the healthy-28d calves.

Analysis at the strain level revealed numeric differences between healthy animals and animals that developed BRD for Mannheimia haemolytica non-serotype A1 str. PHL213 ( $P=0.11$; Figure 6$)$. The relative abundance of this specific serotype (expressed as a proportion of all Mannheimia haemolytica serotypes) was increased in BRD-14d calves $(0.44 \pm 0.14)$ and BRD-28d calves $(0.49 \pm 0.11)$ compared with healthy-14d calves $(0.35 \pm$ 0.12 ), and decreased compared with healthy-28d calves 

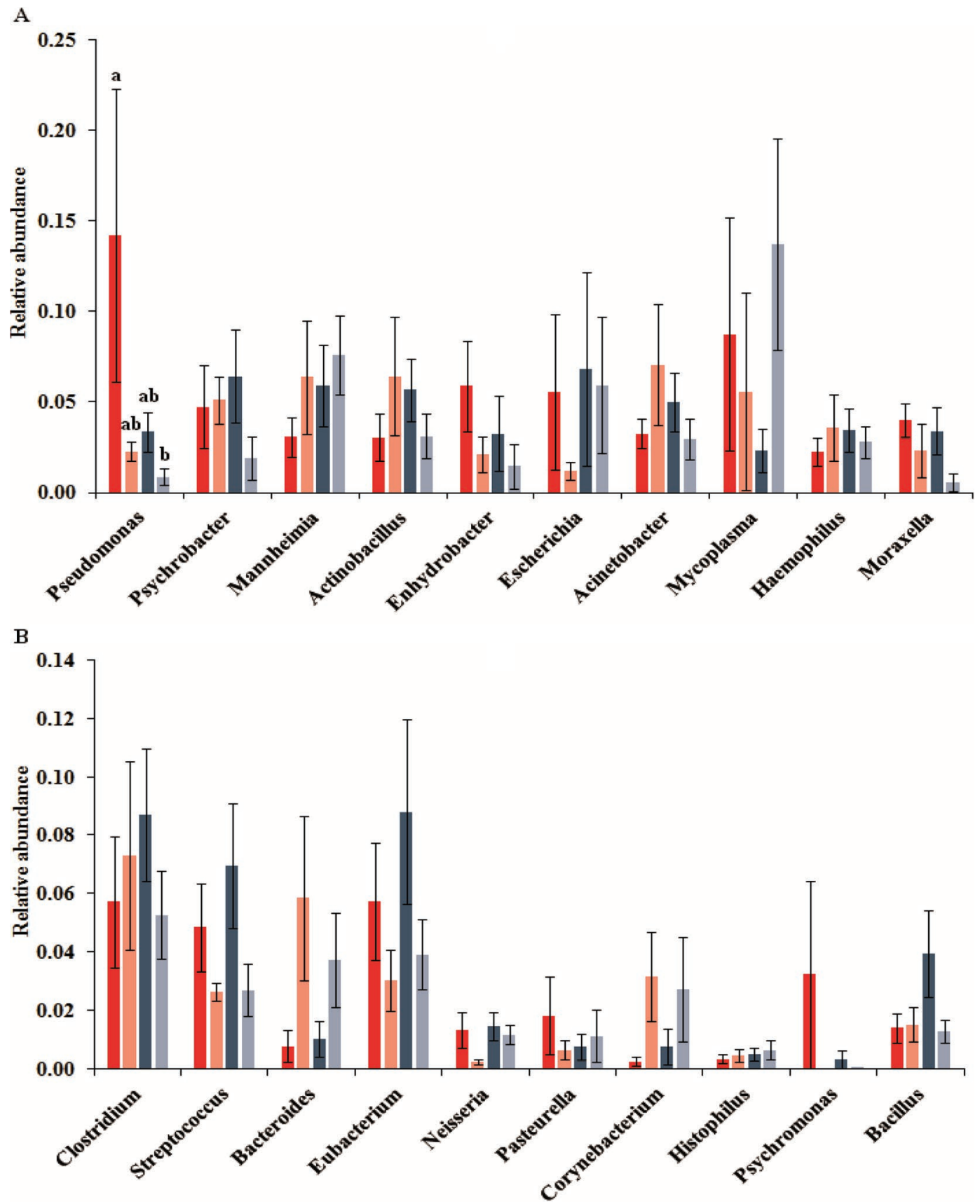

Figure 2. Relative abundance of the 20 most abundant genera (the 10 most abundant in panel A, and the remainder in panel B) in deep nasopharyngeal swab samples obtained from healthy calves (right 2 bars for each genus; blue) and calves that developed bovine respiratory disease (left 2 bars for each genus; red) at d 14 (darker shades) and d 28 (lighter shades) of life. Error bars represent SEM. Different letters indicate Tukey-adjusted significant difference between groups $(P<0.05)$. Color version available online.

(0.53 \pm 0.09$)$. Relative abundances of Mannheimia haemolytica serotype A1 str. PHL213 were also increased in BRD-14d calves $(0.23 \pm 0.07)$ and BRD-28d calves $(0.34 \pm 0.09)$ compared with healthy-14d calves $(0.15$ $\pm 0.05)$, and decreased compared with healthy-28d calves $(0.37 \pm 0.08$; Figure 6$)$.

\section{Functional Profile}

In addition to a taxonomic profile, shotgun sequencing provides information about samples' functional profile. The relative abundance of the 20 most abundant functional features is shown in Figure 7. The relative 
abundance of some functional features tended to be numerically increased in samples from BRD calves compared with healthy calves (amino acids and derivatives: $P=0.87$; carbohydrates: $P=0.84$; cell division and cell cycle: $P=0.95$; cell wall and capsule: $P=$ 0.99; clustering-based subsystems: $P=0.61$; cofactors, vitamins, prosthetic groups, pigments: $P=0.88$; DNA metabolism: $P=0.97$; dormancy and sporulation: $P=$ 0.17 ; fatty acids and isoprenoids: $P=0.94$; iron acquisition and metabolism: $P=0.90$; membrane transport:
$P=0.95$; metabolism and aromatic compounds: $P=$ 0.09 ; miscellaneous: $P=0.88$; motility and chemotaxis: $P=0.83$; nitrogen metabolism: $P=0.93$; nucleosides and nucleotides: $P=0.87$; phages, prophages, transposable elements, and plasmids: $P=0.88$; phosphorus metabolism: $P=0.67$; photosynthesis: $P=0.82$; potassium metabolism: $P=0.99$; protein metabolism: $P=$ 0.73 ; RNA metabolism: $P=0.81$; regulation and cell signaling: $P=0.58$; secondary metabolism: $P=0.93$; stress metabolism: $P=0.76$; sulfur metabolism: $P=$
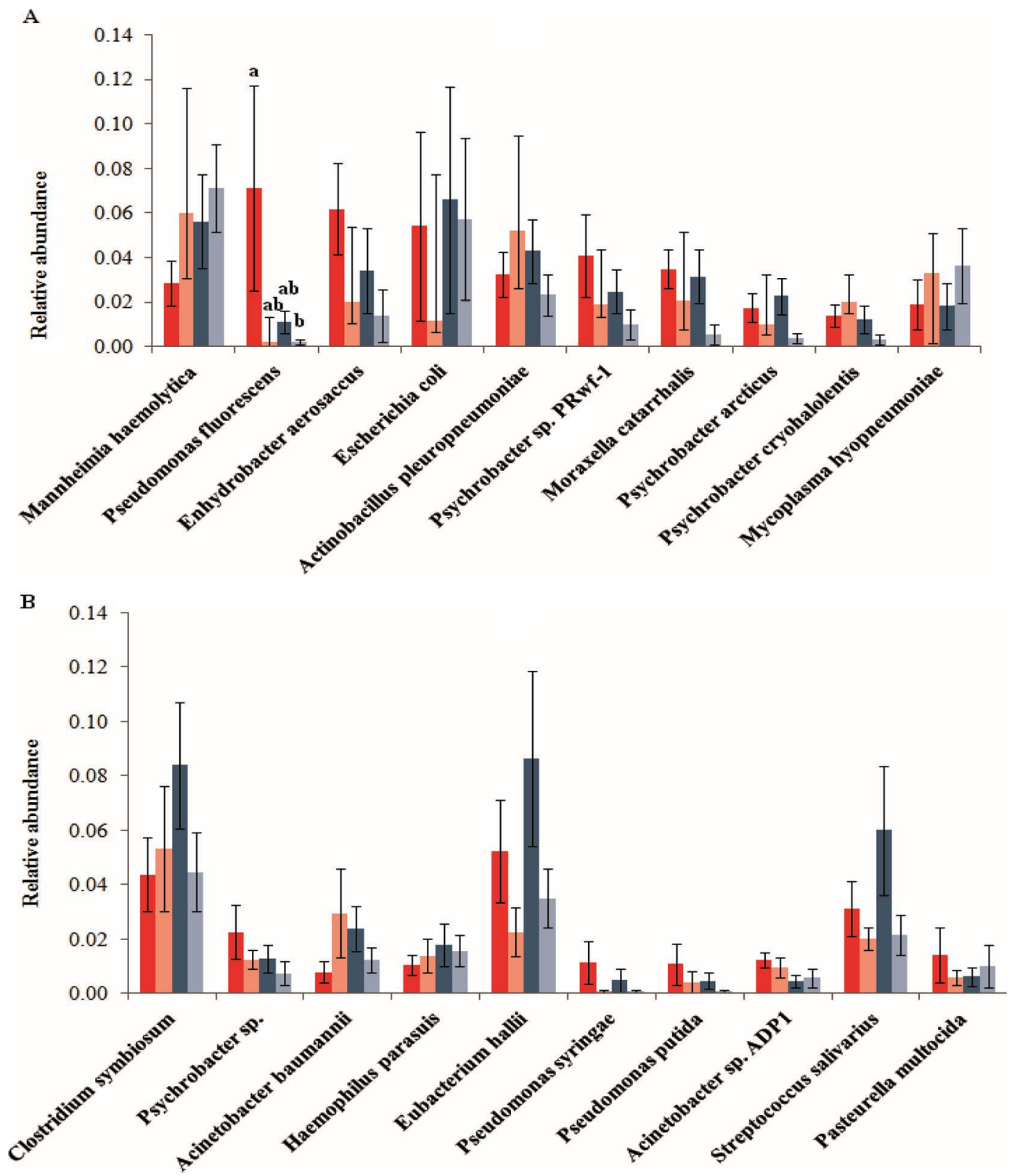

Figure 3. Relative abundance of the 20 most abundant species (the 10 most abundant in panel A, and the remainder in panel B) in deep nasopharyngeal swab samples obtained from healthy calves (right 2 bars for each species; blue) and calves that developed bovine respiratory disease (left 2 bars for each species; red) at d 14 (darker shades) and d 28 (lighter shades) of life. Error bars represent SEM. Different letters indicate Tukey-adjusted significant difference between groups $(P<0.05)$. Color version available online. 


\section{Species}

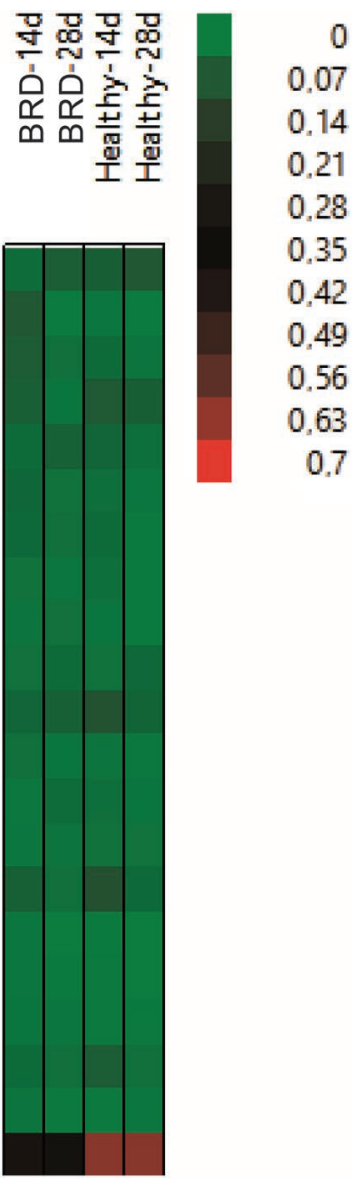

calves $(0.10 \pm 0.08)$ compared to healthy calves $(P=$ $0.57)$. Resistance to fluoroquinolones was numerically increased in healthy-28d calves $(0.38 \pm 0.13)$ and BRD$28 \mathrm{~d}$ calves $(0.29 \pm 0.16 ; P=0.57)$. Methicillin-resistant staphylococcus $(P=0.29)$ and copper homeostasis features $(P=0.66)$ were both numerically increased in BRD-28d (0.19 $\pm 0.16 ; 0.04 \pm 0.04$, respectively). We detected beta-lactamase $(P=0.55)$ only in healthy-14d calves $(0.01 \pm 0.01)$. Cadmium resistance was numerically increased in BRD-28d calves $(0.08 \pm 0.08)$ compared with the other groups $(P=0.25$; Figure 9$)$.

\section{DISCUSSION}

To better understand the upper respiratory tract metagenome in dairy calves, we used shotgun sequencing of deep nasopharyngeal samples and characterized the differences between healthy animals and animals with BRD at 14 and $28 \mathrm{~d}$ of life. We selected these 2 time points mainly because all BRD cases in the Lima et al. (2016) study were diagnosed between d 14 and 21 of life. Our data revealed a remarkable complexity in the bacterial ecology of both healthy and BRD-affected calves. We detected Moraxella catarrhalis, a species known to be associated with upper respiratory tract disease in humans, in all groups, particularly in BRD calves, although this difference was not statistically significant. In our functional profile analysis, we observed a greater abundance of genes that were resistant to cobalt-zinc-cadmium in BRD-14d samples, which could indicate difficulties in treatment of these animals, given that the presence of these genes has been associated with increased resistance to antibiotics.

Our study could have been more informative had we been able to obtain bronchoalveolar lavage samples (at least at the second sampling time point), which would have allowed us to study the lung metagenome in addition to that of the upper respiratory tract. However, our study was conducted on a commercial dairy farm, and it was not possible for us to secure the owner's consent for such a sampling approach. The small number of enrolled calves represents another limitation of our study. Although shotgun metagenomic studies are commonly conducted in a small number of subjects (because the cost of conducting such studies remains high) this limitation should be acknowledged, and the results presented here should be interpreted with caution. It should also be noted that our study was conducted on a single farm; a larger-scale, multi-farm study could have been more informative. Sampling time points were fixed (14 and $28 \mathrm{~d}$ of life) and dictated by the Lima et al. (2016) study design. This represents another limitation of our study, because calves developed BRD and 
Table 1. Number (\%) of calves with a positive detection of bovine respiratory disease (BRD) bacterial pathogens according to health status (healthy and BRD) and sampling time (14 and $28 \mathrm{~d})$

\begin{tabular}{|c|c|c|c|c|c|}
\hline Pneumonic pathogen & BRD-14d & BRD-28d & Healthy-14d & Healthy-28d & Total \\
\hline Histophilus somni & $4(66.7)$ & $4(66.7)$ & $4(40.0)$ & $4(40.0)$ & $16(50.0)$ \\
\hline
\end{tabular}

were treated with antibiotics at different time points (between 14 and $28 \mathrm{~d}$ of life).

Calves that were diagnosed with BRD in our study received antibiotic therapy, and systemic antibiotic therapy in calves should affect the microbiome of the upper respiratory tract. The post-treatment microbiome is likely a reflection of the microorganisms involved in the disease process and the effect of antimicrobial treatment. We have recently shown that parenteral administration of antibiotics has a temporal effect on neonatal calves' fecal microbiome, and this should also be the case for the upper respiratory tract microbiome (Oultram et al., 2015). Our study was conducted on a commercial dairy farm, and farm protocols could not be modified solely for this study. Additionally, BRD is commonly caused by bacterial infection, and neglecting to treat sick calves with the proper antibiotic therapy could be considered inhumane and may not have been approved by the Cornell Institutional Animal Care and Use Committee. Differences between samples obtained at different time points could also be due to the evolution of the nasopharyngeal microbiota. Timsit et al. (2016) recently reported that the nasopharyngeal microbiota of beef cattle underwent a profound evolution from weaning until arrival at a feedlot and from arrival to d 40.

Analysis at the phylum level detected 22 phyla, of which Proteobacteria, Firmicutes, Actinobacteria, Bacteroidetes, and Tenericutes were the most abundant. Our results were in agreement with those of Holman et al. (2015a) who, studying the nasopharyngeal microbiome of healthy and BRD calves, detected 22 phyla, of which Proteobacteria and Firmicutes were the major ones. Studies in pigs (Lowe et al., 2011) and children (Bogaert et al., 2011) revealed similar results at the phylum level. Conversely, Tenericutes dominated the

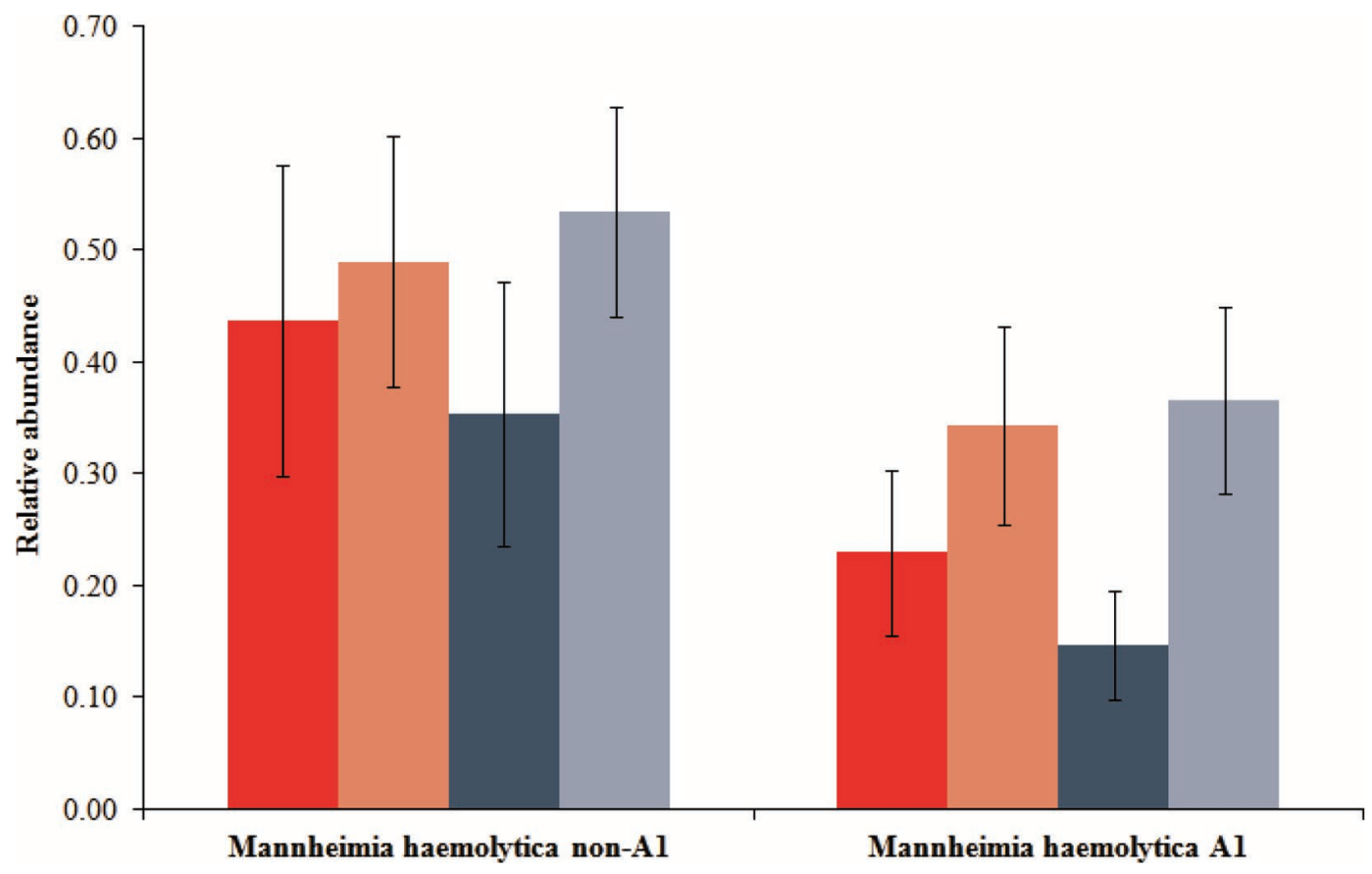

Figure 5. Relative abundance of the most common bacteria related to bovine respiratory disease in deep nasopharyngeal swab samples obtained from healthy calves (right 2 bars in each group; blue) and calves that developed bovine respiratory disease (left 2 bars in each group; red) at d 14 (darker shades) and d 28 (lighter shades) of life. Error bars represent SEM. Statistically significant differences were not observed. Color version available online. 


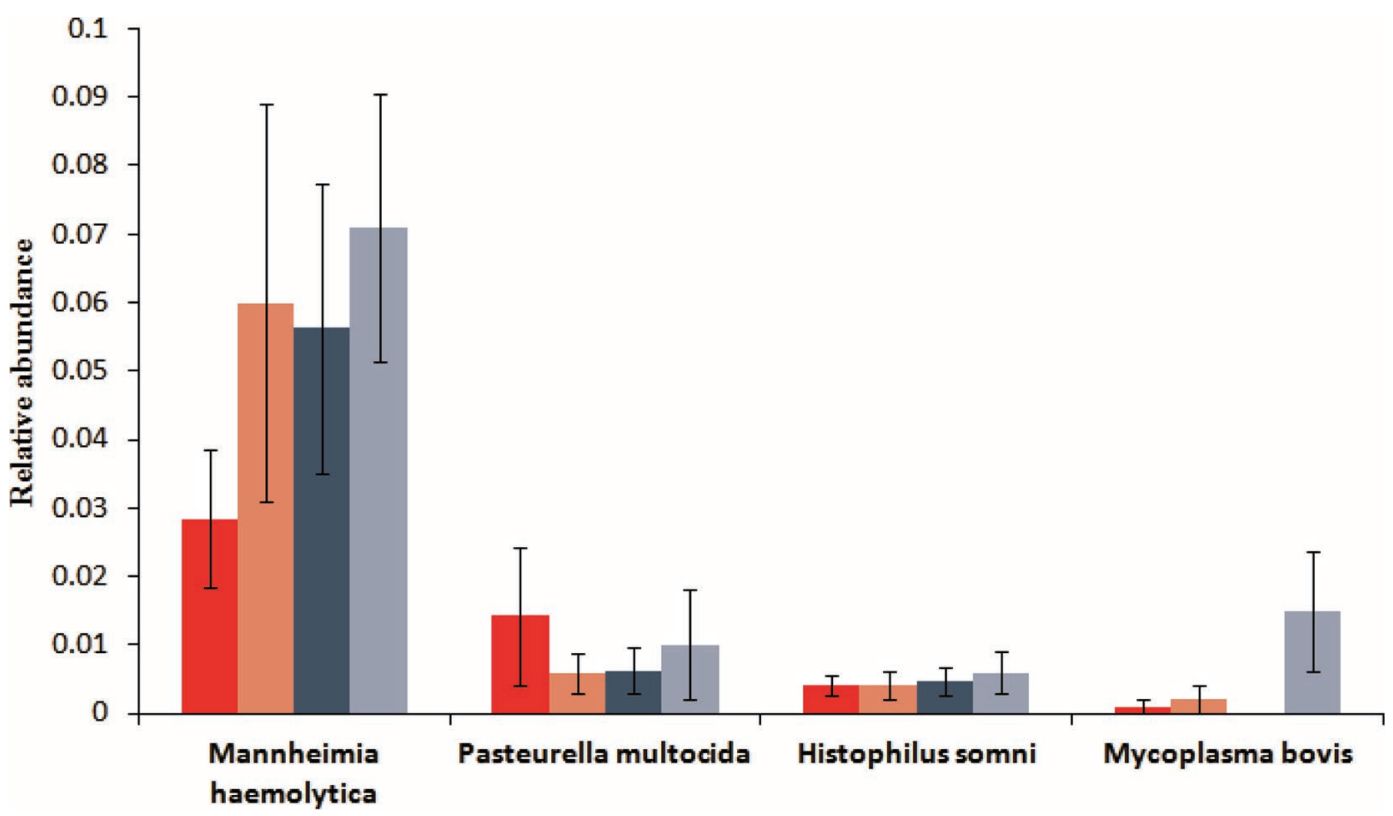

Figure 6. Relative abundance of Mannheimia haemolytica non-A1 and Mannheimia haemolytica A1 in deep nasopharyngeal swab samples obtained from healthy calves (right 2 bars in each group; blue) and calves that developed bovine respiratory disease (left 2 bars in each group; red) at d 14 (darker shades) and d 28 (lighter shades) of life. Error bars represent SEM. Statistically significant differences were not observed. Color version available online.

nasopharyngeal microbiota of beef cattle from weaning to $40 \mathrm{~d}$ after their arrival at a feedlot (Timsit et al., 2016).

Analysis at the genus level revealed 20 major genera that belonged to the aforementioned 5 phyla. Among them, we detected significant differences in the relative abundance of Pseudomonas spp. between groups of calves. Pseudomonas fluorescens was the species responsible for this significant difference, being more abundant in BRD-14d calves. A reduction in its abundance in BRD-28d calves could be associated with antibiotic treatment. Pseudomonas fluorescens has been associated with the production of an antifungal lipopetide for the biocontrol of plant pathogenic fungus (Nielsen et al., 2000). In humans, urinary tract infections (Von Graevenitz and Weinstein, 1971), bacteremia in oncology patients (Hsueh et al., 1998), and a possible role in intestinal mucosal damage in Crohn's disease (Wei et al., 2002; Scales et al., 2014) have all been attributed to Pseudomonas fluorescens. Although Pseudomonas fluorescens has been identified by culture-independent and culture-dependent techniques in broncheoalveolar lavage fluid (Bahrani-Mougeot et al., 2007) and tracheal aspirates (Redding and McWalter, 1980) in patients with pneumonia, its role in respiratory disease remains unclear. In ruminants, Pseudomonas fluorescens has not been reported as a causative agent of any infections.

Mannheimia haemolytica, Mycoplasma bovis, Histophilus somni, and Pasteurella multocida are the most commonly reported bacteria in pneumonia cases in cattle (Rice et al., 2007; Griffin et al., 2010). In the present study, Mannheimia haemolytica was the most abundant in all groups and was detected in most of the studied calves. Its relative abundance was increased in both groups of calves, particularly among healthy-28d calves. Mannheimia haemolytica is an upper respiratory tract commensal that can become pathogenic under certain circumstances (Griffin et al., 2010). Several studies have reported its presence in the respiratory tract of both healthy and BRD calves, most of which observed an association between this bacterium and pneumonia (Angen et al., 2009; Francoz et al., 2015; Holman et al., 2015a). Analysis of Mannheimia haemolytica strains showed the presence of serotype A2 in all groups. This is the predominant strain detected in healthy calves (Rice et al., 2007), and it is associated with pneumonia in sheep (Lawrence et al., 2010). On the other hand, pneumonia in cattle is highly associated with serotypes A1 and A6 (Griffin, 2010). Interestingly, we detected numeric differences in the relative abundance of Mannheimia haemolytica serotype A1 str. PHL 213 between healthy and BRD calves, in accordance with previous research (Purdy et al., 1997; Al-Ghamdi et al., 2000; Griffin et al., 2010).

Although Mycoplasma bovis is commonly reported as a causative agent of pneumonia in calves (Kusiluka et al., 2000; Gagea et al., 2006), we could only detect it in very low relative abundances. Mycoplasma bovis 


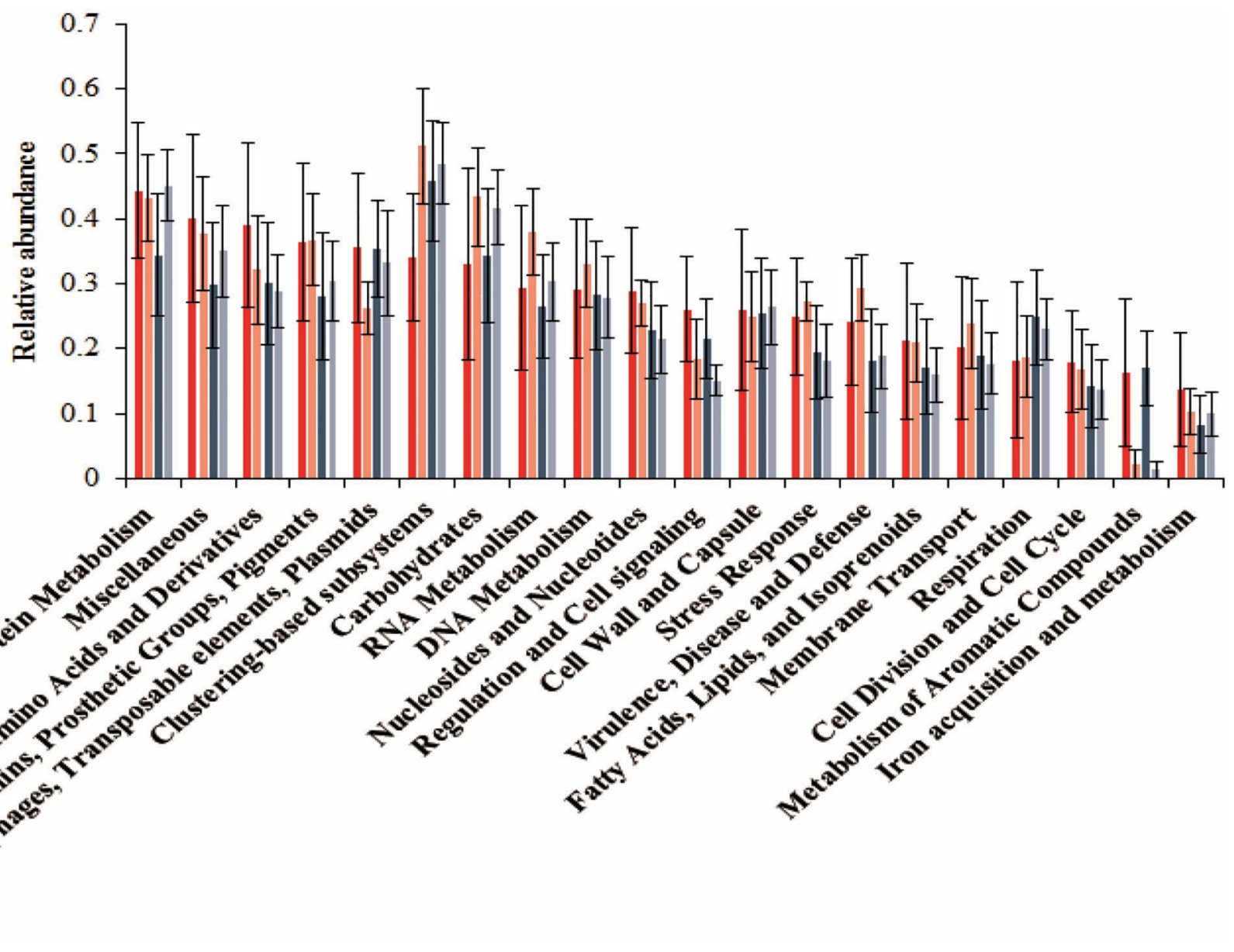

Figure 7. Relative abundance of the 20 most abundant functional profile features in deep nasopharyngeal swab samples obtained from healthy calves (right 2 bars in each group; blue) and calves that developed bovine respiratory disease (left 2 bars in each group; red) on d 14 (darker shades) and d 28 (lighter shades) of life. Error bars represent SEM. Statistically significant differences were not observed. Color version available online.

sequences were detected in 1 of 6 BRD-14d calves, 1 of 6 BRD-28d calves, and 4 of 10 healthy-28d calves. Mycoplasma bovis sequences were absent from all healthy-14d calves. The rather low relative abundance of Mycoplasma bovis and the presence of other pneumonia-associated relevant bacteria support the hypothesis that Mycoplasma bovis is an opportunistic pathogen (Rosendal and Martin, 1986) and part of calves' normal upper respiratory tract microbiome.

Histophilus somni is a commensal microorganism that belongs to the nasopharyngeal microbiome of calves (Griffin et al., 2010), and like other bacteria that reside in the upper respiratory tract, becomes pathogenic after changes in the upper respiratory tract environment. In the present study, Histophilus somni was less abundant than Mannheimia haemolytica, Mycoplasma bovis, or Pasteurella multocida.

Pasteurella multocida was 1 of the 20 most abundant bacteria detected in the deep nasal swab samples. It was detected in both healthy and sick groups, but it was not identified in all samples. We detected Pasteurella multocida in BRD calves in slightly higher abundance, particularly at $14 \mathrm{~d}$ of life; however, we detected no statistically significant differences between groups. Several studies detected Pasteurella multocida in the respiratory tract of calves, using culture-dependent and culture-independent techniques; in most of them, this bacterium was highly prevalent (Angen et al., 2009; Jamali et al., 2014; Holman et al., 2015b).

Moraxella catarrhalis is a well-known gram-negative pathogen associated with respiratory disease in humans (Verduin et al., 2002; Murphy and Parameswaran, 2009), particularly in children (Sy and Robinson, 2010). Although it is described as an exclusive pathogen of the upper respiratory tract in humans (Murphy and Parameswaran, 2009), Moraxella catarrhalis was isolated from lungs of BRD calves, and its presence has also been demonstrated in lung lesions from the same calves 


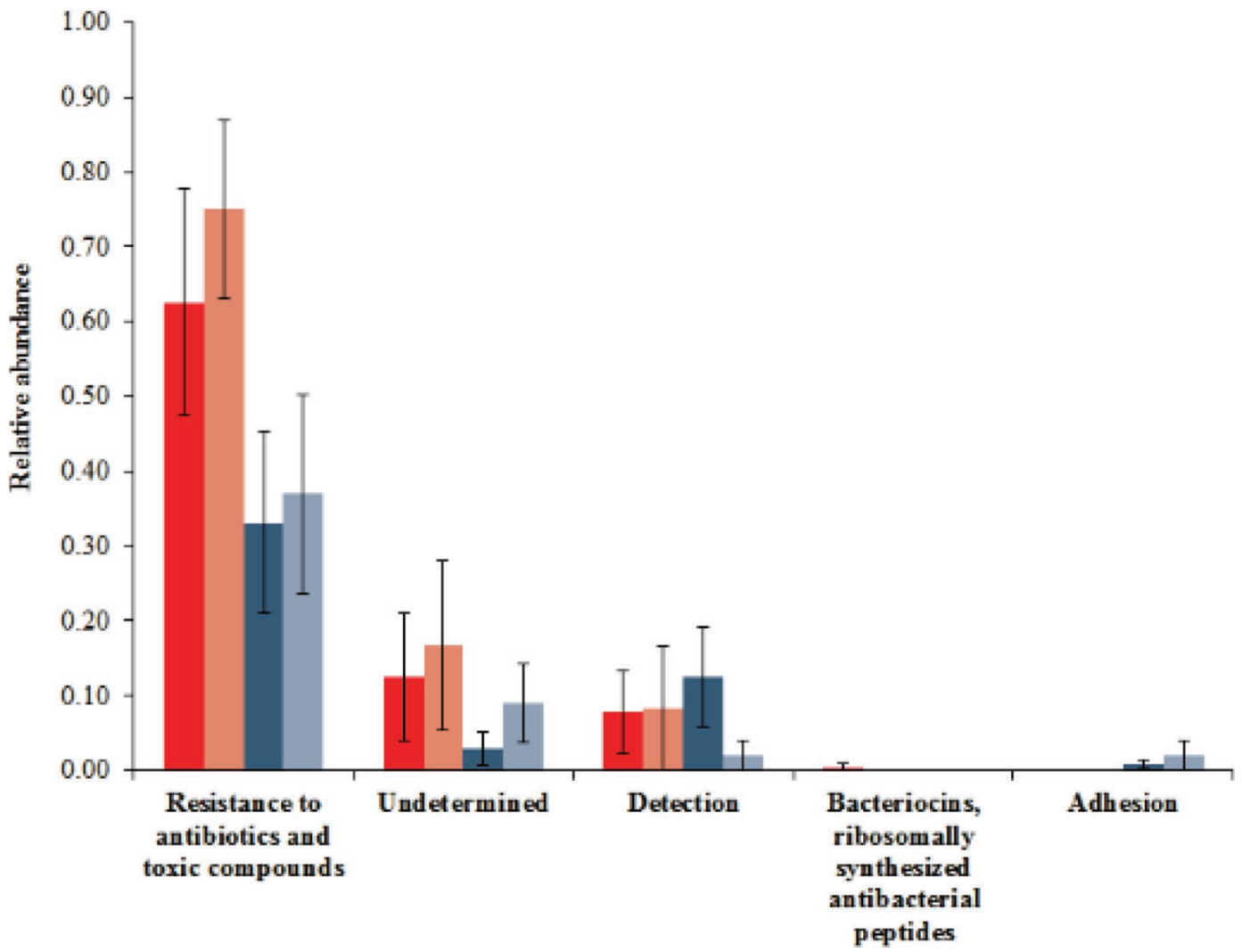

Figure 8. Normalized abundance distribution of virulence and disease and defense factors in deep nasopharyngeal swab samples obtained from healthy calves (right 2 bars in each group; blue) and calves that developed bovine respiratory disease (left 2 bars in each group; red) at d 14 (darker shades) and d 28 (lighter shades) of life. Error bars represent SEM. Statistically significant differences were not observed. Color version available online.

by immunohistology in the study conducted by Nagai et al., (1995). Recently, Moraxella spp. was detected in the nasopharyngeal tract of calves in a feedlot (Holman et al., 2015a), but, the species was not determined and the health status of the calves was not reported.

Actinobacillus pleuropneumoniae, Mycoplasma hyopneumoniae, and Haemophilus parasuis were among the 20 most abundant bacteria detected in deep nasopharyngeal swab samples. These species are well-known pathogens associated with swine respiratory disease (Bossé et al., 2002; Nedbalcova et al., 2006; Sibila et al., 2009). Although Actinobacillus pleuropneumoniae is considered an exclusive pathogen of the swine respiratory tract, this species has been isolated in cattle, deer, and lambs (Desrosiers and Moore, 1998). The potential role of these fastidious bacteria in BRD may warrant further investigation.

Functional profiling showed numeric differences between healthy calves and calves that developed BRD, the latter having increased abundances of features related to resistance to antibiotics and toxic compounds. Cadmium-zinc-cobalt resistance has been observed in bacteria such as Staphylococcus aureus and Escherichia coli and is related to proteins such as cadCA, cadB, czcCBA, and cnr, depending on the bacteria (Nies, 1992). Five mechanisms of metal resistance have been described (Choudhury and Srivastava, 2001): exclusion by permeable barrier, exclusion by active transport, intra- and extracellular physical sequestration, and transformation and detoxification. Studies reported heavy metals as a source of coselection of antibiotic resistance (Pettibone et al., 1996; Stepanauskas et al., 2005; Seiler and Berendonk, 2012). Coselection occurs due to physiological (cross-resistance) and genetic (coresistance) mechanisms. The first provides resistance to more than 1 antibiotic agent (drugs and heavy metal) using mechanisms such as multidrug efflux pumps (Baker-Austin et al., 2006; Martinez et al., 2009) and the second is related to 2 or more genetically linked resistance genes (Chapman, 2003). In the present study, genes related to heavy-metal resistance were increased in BRD calves compared to healthy calves, and could imply difficulties in the treatment of diseased calves, given that studies have highlighted the importance of coselection and heavy-metal resistance for antibiotic resistance (Chapman, 2003). Genes associated with an- 


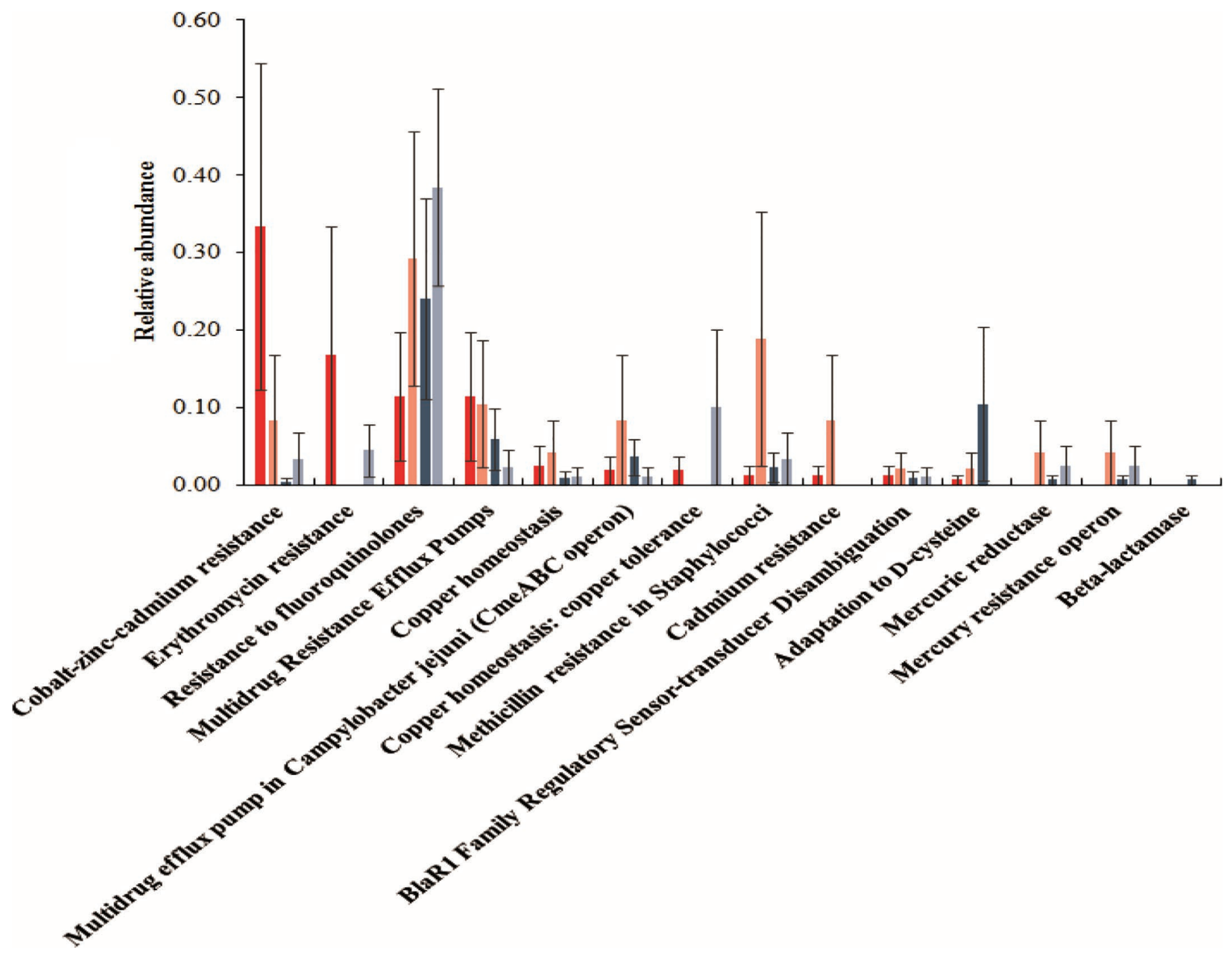

Figure 9. Normalized abundance distribution of resistance to antibiotics and toxic compound features in deep nasopharyngeal swab samples obtained from healthy calves (right 2 bars in each group; blue) and calves that developed bovine respiratory disease (left 2 bars in each group; red) at d 14 (darker shades) and d 28 (lighter shades) of life. Error bars represent SEM. Statistically significant differences were not observed. Color version available online.

tibiotic resistance were also more abundant in samples obtained from BRD calves.

\section{CONCLUSIONS}

In this study, Mannheimia haemolytica and Pasteurella multocida were among the 20 most abundant bacteria found in healthy and BRD calves. Moraxella catarrhalis, an important bacterium for human pneumonia, was detected in both groups, particularly in BRD calves. The importance of Moraxella catarrhalis for calves' respiratory disease remains elusive. Genes resistant to cobalt-zinc-cadmium, observed mostly in BRD calves, might be associated with difficulties in treatment with antibiotics.

\section{ACKNOWLEDGMENTS}

We gratefully acknowledge the São Paulo Research Foundation (FAPESP) for providing funding (protocol \# 2015/02947-0).

\section{REFERENCES}

Aebi, M., B. H. van den Borne, A. Raemy, A. Steiner, P. Pilo, and M. Bodmer. 2015. Mycoplasma bovis infections in Swiss dairy cattle: A clinical investigation. Acta Vet. Scand. 57:10. https://doi. org/10.1186/s13028-015-0099-x.

Al-Ghamdi, G. M., T. R. Ames, J. C. Baker, R. Walker, C. C. Chase, G. H. Frank, and S. K. Maheswaran. 2000. Serotyping of Mannheimia (Pasteurella) haemolytica isolates from the upper Midwest United States. J. Vet. Diagn. Invest. 12:576-578. https:// doi.org/10.1177/104063870001200617. 
Angen, O., J. Thomsen, L. E. Larsen, J. Larsen, B. Kokotovic, P. M. H. Heegaard, and J. M. D. Enemark. 2009. Respiratory disease in calves: Microbiological investigations on trans-tracheally aspirated bronchoalveolar fluid and acute phase protein response. Vet. Microbiol. 137:165-171. https://doi.org/10.1016/j.vetmic.2008.12.024.

Bahrani-Mougeot, F. K., B. J. Paster, S. Coleman, S. Barbuto, M. T. Brennan, J. Noll, T. Kennedy, P. C. Fox, and P. B. Lockhart. 2007. Molecular analysis of oral and respiratory bacterial species associated with ventilator-associated pneumonia. J. Clin. Microbiol 45:1588-1593. https://doi.org/10.1128/JCM.01963-06.

Baker-Austin, C., M. S. Wright, R. Stepanauskas, and J. V. McArthur. 2006. Co-selection of antibiotic and metal resistance. Trends Microbiol. 14:176-182. https://doi.org/10.1016/j.tim.2006.02.006.

Bogaert, D., B. Keijser, S. Huse, J. Rossen, R. Veenhoven, E. van Gils, J. Bruin, R. Montijn, M. Bonten, and E. Sanders. 2011. Variability and diversity of nasopharyngeal microbiota in children: A metagenomic analysis. PLoS One 6:e17035. https://doi. org/10.1371/journal.pone.0017035.

Bosch, A. A. T. M., G. Biesbroek, K. Trzcinski, E. A. M. Sanders, and D. Bogaert. 2013. Viral and bacterial interactions in the upper respiratory tract. PLoS Pathog. 9. https://doi.org/10.1371/ journal.ppat.1003057.

Bossé, J. T., H. Janson, B. J. Sheeran, A. J. Beddek, A. N. Rycroft, J. S. Kroll, and P. R. Langford. 2002. Actinobacillus pleuropneumoniae: Pathobiology and pathogenesis of infection. Microbes Infect. 4:225-235. https://doi.org/10.1016/S1286-4579(01)01534-9

Cernicchiaro, N., B. J. White, D. G. Renter, and A. H. Babcock. 2013 Evaluation of economic and performance outcomes associated with the number of treatments after an initial diagnosis of bovine respiratory disease in commercial feeder cattle. Am. J. Vet. Res. 74:300-309. https://doi.org/10.2460/ajvr.74.2.300.

Chapman, J. S. 2003. Disinfectant resistance mechanisms, cross-resistance, and co-resistance. Int. Biodeterior. Biodegradation 51:271276. https://doi.org/10.1016/S0964-8305(03)00044-1.

Choudhury, R., and S. Srivastava. 2001. Zinc resistance mechanisms in bacteria. Curr. Sci. 81:768-775.

Desrosiers, R., and C. Moore. 1998. Indirect transmission of Actinobacillus pleuropneumoniae. Swine Health Prod. 6:263-265.

Francoz, D., S. Buczinski, A. M. Belanger, G. Forte, O. Labrecque, D. Tremblay, V. Wellemans, and J. Dubuc. 2015. Respiratory pathogens in Quebec dairy calves and their relationship with clinical status, lung consolidation, and average daily gain. J. Vet. Intern. Med. 29:381-387. https://doi.org/10.1111/jvim.12531.

Gagea, M. I., K. G. Bateman, R. A. Shanahan, T. van Dreumel, B. J. McEwen, S. Carman, M. Archambault, and J. L. Caswell. 2006 Naturally occurring Mycoplasma bovis-associated pneumonia and polyarthritis in feedlot beef calves. J. Vet. Diagn. Invest. 18:29-40. https://doi.org/10.1177/104063870601800105.

Gershwin, L. J., A. L. Van Eenennaam, M. L. Anderson, H. A. McEligot, M. X. Shao, R. Toaff-Rosenstein, J. F. Taylor, H. L. Neibergs, and J. Womack. 2015. Single pathogen challenge with agents of the bovine respiratory disease complex. PLoS One 10:e0142479. https://doi.org/10.1371/journal.pone.0142479.

Murphy, T. F., and G. I. Parameswaran. 2009. Moraxella catarrhalis, a human respiratory tract pathogen. Clin. Infect. Dis. 49:124-131. https://doi.org/10.1086/599375.

Griffin, D. 1997. Economic impact associated with respiratory disease in beef cattle. Vet. Clin. North Am. Food Anim. Pract. 13:367-377.

Griffin, D. 2010. Bovine pasteurellosis and other bacterial infections of the respiratory tract. Vet. Clin. North Am. Food Anim. Pract. 26:57-71. https://doi.org/10.1016/j.cvfa.2009.10.010.

Griffin, D., M. M. Chengappa, J. Kuszak, and D. S. McVey. 2010. Bacterial pathogens of the bovine respiratory disease complex. Vet. Clin. North Am. Food Anim. Pract. 26:381-394. https://doi org/10.1016/j.cvfa.2010.04.004.

Holman, D. B., T. McAllister, E. Topp, A.-D. G. Wright, and T. W. Alexander. 2015a. The nasopharyngeal microbiota of feedlot cattle that develop bovine respiratory disease. Vet. Microbiol. https:// doi.org/10.1016/j.vetmic.2015.07.031
Holman, D. B., E. Timsit, and T. W. Alexander. 2015b. The nasopharyngeal microbiota of feedlot cattle. Sci. Rep. https://doi. org/10.1038/srep15557.

Hsueh, P.-R., L.-J. Teng, H.-J. Pan, Y.-C. Chen, C.-C. Sun, S.-W. Ho, and K.-T. Luh. 1998. Outbreak of Pseudomonas fluorescens bacteremia among oncology patients. J. Clin. Microbiol. 36:2914-2917.

Jamali, H., M. Rezagholipour, S. Fallah, and A. Dadrasnia. 2014. Prevalence, characterization and antibiotic resistance of Pasteurella multocida isolated from bovine respiratory infection. Vet. J. 202:381-383. https://doi.org/10.1016/j.tvj1.2014.07.024.

Kusiluka, L. J., B. Ojeniyi, and N. F. Friis. 2000. Increasing prevalence of Mycoplasma bovis in Danish cattle. Acta Vet. Scand. 41:139-146.

Lawrence, P. K., W. Kittichotirat, R. E. Bumgarner, J. E. McDermott, D. R. Herndon, D. P. Knowles, and S. Srikumaran. 2010. Genome sequences of Mannheimia haemolytica serotype A2: Ovine and bovine isolates. J. Bacteriol. 192:1167-1168. https://doi. org/10.1128/JB.01527-09

Lima, S. F., A. G. V. Teixeira, C. H. Higgins, F. S. Lima, and R. C. Bicalho. 2016. The upper respiratory tract microbiome and its potential role in bovine respiratory disease and otitis media. Sci. Rep. 6:29050 https://doi.org/10.1038/srep29050.

Lowe, B. A., T. L. Marsh, N. Isaacs-Cosgrove, R. N. Kirkwood, M. Kiupel, and M. H. Mulks. 2011. Microbial communities in the tonsils of healthy pigs. Vet. Microbiol. 147:346-357. https://doi. org/10.1016/j.vetmic.2010.06.025.

Martinez, J. L., M. B. Sánchez, L. Martínez-Solano, A. Hernandez, L. Garmendia, A. Fajardo, and C. Alvarez-Ortega. 2009. Functional role of bacterial multidrug efflux pumps in microbial natural ecosystems. FEMS Microbiol. Rev. 33:430-449. https://doi. org/10.1111/j.1574-6976.2008.00157.x.

Nagai, M., T. Ozawa, N. Ishikawa, H. Komae, Y. Hayakawa, and M. Nakazuwa. 1995. Isolation of Moraxella catarrhalis from calves with pneumonia. J. Jpn. Vet. Med. Assoc. 48:319-322. https:// doi.org/10.12935/jvma1951.48.319.

Nedbalcova, K., P. Satran, Z. Jaglic, R. Ondriasova, and Z. Kucerova. 2006. Haemophilus parasuis and Glässer's disease in pigs: A review. Vet. Med. 51:168-179.

Nielsen, T. H., C. Thrane, C. Christophersen, U. Anthoni, and J. Sorensen. 2000. Structure, production characteristics and fungal antagonism of tensin-A new antifungal cyclic lipopeptide from Pseudomonas fluorescens strain 96.578. J. Appl. Microbiol. 89:9921001. https://doi.org/10.1046/j.1365-2672.2000.01201.x.

Nies, D. H. 1992. Resistance to cadmium, cobalt, zinc, and nickel in microbes. Plasmid 27:17-28

Oultram, J., E. Phipps, A. G. V. Teixeira, C. Foditsch, M. L. Bicalho, V. S. Machado, R. C. Bicalho, and G. Oikonomou. 2015. Effects of antibiotics (oxytetracycline, florfenicol or tulathromycin) on neonatal calves' faecal microbial diversity. Vet. Rec. https://doi. org/10.1136/vr.103320

Pettibone, G. W., J. P. Mear, and B. M. Sampsell. 1996. Incidence of antibiotic and metal resistance and plasmid carriage in Aeromonas isolated from brown bullhead (Ictalurus nebulosus). Lett. Appl. Microbiol. 23:234-240. https://doi.org/10.1111/j.1472-765X.1996. tb00073.x.

Purdy, C. W., R. H. Raleigh, J. K. Collins, J. L. Watts, and D. C. Straus. 1997. Serotyping and enzyme characterization of Pasteurella haemolytica and Pasteurella multocida isolates recovered from BRD lungs of stressed feeder calves. Curr. Microbiol. 34:244-249. https://doi.org/10.1007/s002849900177.

Redding, P. J., and P. W. McWalter. 1980. Pseudomonas fluorescens cross-infection due to contaminated humidifier water. BMJ 281:275. https://doi.org/10.1136/bmj.281.6235.275.

Rice, J. A.. L. Carrasco-Medina, D. C. Hodgins, and P. E. Shewen. 2007. Mannheimia haemolytica and bovine respiratory disease. Anim. Health Res. Rev. 8:117-128. https://doi.org/10.1017/ S1466252307001375.

Rosendal, S., and S. Martin. 1986. The association between serological evidence of mycoplasma infection and respiratory disease in feedlot calves. Can. J. Vet. Res. 50:179-183. 
Scales, B. S., R. P. Dickson, J. J. LiPuma, and G. B. Huffnagle. 2014. Microbiology, genomics, and clinical significance of the Pseudomonas fluorescens species complex, an unappreciated colonizer of humans. Clin. Microbiol. Rev. 27:927-948. https://doi.org/10.1128/ CMR.00044-14

Seiler, C., and T. U. Berendonk. 2012. Heavy metal driven co-selection of antibiotic resistance in soil and water bodies impacted by agriculture and aquaculture. Front. Microbiol. 3:399. https://doi. org/10.3389/fmicb.2012.00399.

Sharpton, T. J. 2014. An introduction to the analysis of shotgun metagenomic data. Front. Plant Sci. 5:209. https://doi. org/10.3389/fpls.2014.00209.

Sibila, M., M. Pieters, T. Molitor, D. Maes, F. Haesebrouck, and F. Segalés. 2009. Current perspectives on the diagnosis and epidemiology of Mycoplasma hyopneumoniae infection. Vet. J. 181:221231. https://doi.org/10.1016/j.tvj1.2008.02.020

Stepanauskas, R., T. C. Glenn, C. H. Jagoe, R. C. Tuckfield, A. H. Lindell, and J. V. McArthur. 2005. Elevated microbial tolerance to metals and antibiotics in metal-contaminated industrial environments. Environ. Sci. Technol. 39:3671-3678. https://doi. org/10.1021/es048468f.

Sy, M. G., and J. L. Robinson. 2010. Community-acquired Moraxella catarrhalis pneumonia in previously healthy children. Pediatr. Pulmonol. 45:674-678. https://doi.org/10.1002/ppu1.21243.

Timsit, E., D. B. Holman, J. Hallewell, and T. W. Alexander. 2016. The nasopharyngeal microbiota in feedlot cattle and its role in respiratory health. Anim. Front. 6:44-50. https://doi.org/10.2527/ af.2016-0022.
USDA. 2013. Feedlot 2001. Part IV: Health and Health Management on U.S. Feedlots with a Capacity of 1,000 or more Head. APHIS National Animal Health Monitoring System, Fort Collins, CO.

Verduin, C. M., C. Hol, A. Fleer, H. van Dijk, and A. van Belkum. 2002. Moraxella catarrhalis: From emerging to established pathogen. Clin. Microbiol. Rev. 15:125-144. https://doi.org/10.1128/ CMR.15.1.125-144.2002.

Virtala, A. M., G. D. Mechor, Y. T. Gröhn, and H. N. Erb. 1996a. Morbidity from nonrespiratory diseases and mortality in dairy heifers during the first three months of life. J. Am. Vet. Med. Assoc. 208:2043-2046.

Virtala, A. M., G. D. Mechor, Y. T. Gröhn, and H. N. Erb. 1996b. The effect of calfhood diseases on growth of female dairy calves during the first 3 months of life in New York State. J. Dairy Sci. 79:1040-1049.

Von Graevenitz, A., and J. Weinstein. 1971. Pathogenic significance of Pseudomonas fluorescens and Pseudomonas putida. Yale J. Biol. Med. 44:265-273.

Waltner-Toews, D., S. W. Martin, and A. H. Meek. 1986. The effect of early calfhood health status on survivorship and age at first calving. Can. J. Vet. Res. 50:314-317.

Wei, B., T. Huang, H. Dalwadi, C. L. Sutton, D. Bruckner, and J. Braun. 2002. Pseudomonas fluorescens encodes the Crohn's disease-associated I2 sequence and T-cell superantigen. Infect. Immun. 70:6567-6575. https://doi.org/10.1128/IAI.70.12.65676575.2002 